\title{
Dry matter Production and Partitioning of Cluster Bean (Cyamopsis tetragonaloba (L). taub.) Genotypes (Gum) as Influenced by Plant Density and Bio-Inoculants
}

\author{
Shilpa V. Chogatapur* and H.T. Chandranath \\ Department of Agronomy College of Agriculture, Dharwad, \\ University of Agricultural Sciences, Dharwad-580 005, Karnataka, India \\ *Corresponding author
}

\section{A B S T R A C T}

\begin{tabular}{|l|}
\hline Ke y w or d s \\
AM fungi, \\
Bradyrizhobium, \\
Gum, Leaf area, \\
Leaf area index, \\
PSB.
\end{tabular}

The field experiment was conducted under rainfed condition during late Kharif season of 2014 at Agriculture Resaearch Station Annigeri, University of Agricultural Sciences, Dharwad to study the Response of Clusterbean genotypes (gum) to plant density and bio-inoculants. The experimental field was laid out in split-split plot design with three replications. On the basis of results obtained from present investigation parameters like, total dry matter production per plant, dry matter accumulation in different parts of plant, leaf area and leaf area duration at different growth stages recorded higher with genotype gaurishankar-9 as compared to HG-365. Spacing level of $45 \times 15 \mathrm{~cm}$ recorded higher total dry matter production per plant, dry matter accumulation in different parts of plant, leaf area and leaf area duration at different growth stages. Application of Bradyrizhobium + PSB + AM fungi recorded higher total dry matter production per plant and leaf area.

\section{Introduction}

Clusterbean [Cyamopsis tetragonoloba (L.) Taub] grown mainly kharif season in arid and semi-arid region of tropical India. Multiple uses of the clusterbean make it as an important component of cropping systems of the region. Of late, it has acquired the status of industrial crop because of high glactomanan content in the endosperm of its seed, which has multiple industrial uses and thus a main foreign exchange earner for the area (Rathore et al., 2007). Clusterbean seed is used as a concentrate for animals and for extraction of gum. Seeds of clusterbean contains 22-33\% gum (Choudhary et al., 2014). The gum has its use in several industries, viz. textiles, paper, petroleum, pharmaceuticals, food processing, cosmetics, mining explosives and oil drilling. In India it is cultivated over an area of 51.51 lakh ha with the production of 24.60 lakh tonnes with an average yield of $478.0 \mathrm{~kg}$ per ha (Anon., 2013). India is the leading producer of guar and guar gum in the world and its share is around 80 per cent world production. Bio fertilizers play important role in maintaining the long term soil fertility and sustainability. 
It may increase yield of crops by 10-30 percent (Khandelwal et al., 2012). Recent investigations, in the cultivation of gum guar have demonstrated commercial possibilities of growing guar under rainfed conditions. This has opened up possibilities of growing new cash crops in the region, although it is yet to find acceptance as a part of the cropping system of northern dry zone of Karnataka, but no systematic work has been conducted on this aspect. The research information on cultivable aspects is lacking and also performance of different genotypes and spacing levels, hence, there is a need to study the response of clusterbean genotypes (gum purpose) to plant density and bioinoculants.

\section{Materials and Methods}

The experiment was conducted at Agricultural Research Station, Annigeri, UAS Dharwad during the late kharif seasons of 2014. The soil was clay having initial soil $\mathrm{pH}$ of 7.9 and organic carbon $0.49 \%$ and available N, P and $\mathrm{K}$ of $220,21.87 \mathrm{and} 462 \mathrm{~kg} \mathrm{ha}^{-1}$ respectively. The field was prepared by employing one deep ploughing. The average rainfall of area was $665.9 \mathrm{~mm}$ but during 2014 a rainfall of $771.0 \mathrm{~mm}$ was received. The experiment was laid out in split - split plot design 3 replications. Two genotypes (HG- 365 and Gaurishankar -9), two spacings levels of (30 x $10 \mathrm{~cm}$ and $45 \times 15 \mathrm{~cm}$ ) were allotted to main plot, sub plot and four treatments of Bio inoculants (Bradyrhizobium. PSB, AM fungi and Bradyrhizobium + PSB +AM fungi) were allotted to sub sub plot randomly.

The crop was sown on 15 july and harvested on 17 November. Seeds of cluster bean were treated with biofertilizers, Bradyrhizobium + PSB @ $750 \mathrm{~g} \mathrm{ha}^{-1}$ and AM fungi applied at rate of $50 \mathrm{~kg} \mathrm{ha}^{-1}$. Five random plants were selected from each plot, excluding the border row, for taking observations. Five plants were separated into leaf, stem and reproductive parts and dried in an oven at $70^{\circ} \mathrm{C}$ until a constant weight is obtained. Total dry matter was calculated by adding the dry weights of different plant parts and expressed as grams per plant.

\section{Leaf area $\left(\mathrm{cm}^{2}\right.$ plant $\left.^{-1}\right)$}

The leaf area was worked out by disc method on dry weight basis at 30,60,90 DAS and at harvest as per the procedure suggested by Vivekanandan et al., (1972).

$\mathrm{LA}=\frac{\mathrm{Wa} \times \mathrm{A}}{\mathrm{Wd}}$

Where,

LA $=$ Leaf area $\left(\mathrm{cm}^{2}\right.$ plant $\left.{ }^{-1}\right)$

$\mathrm{Wa}=$ Oven dry weight of all leaves (inclusive of 10 disc weight)

$\mathrm{Wd}=$ Oven dry weight of 10 discs in gram,

$A=$ Area of the $10 \operatorname{discs}\left(\mathrm{cm}^{2}\right)$

\section{Leaf area index (LAI)}

It is defined as an assimilatory surface per unit area of land. Leaf area index was worked out by dividing the leaf area per plant by land area occupied by the plant (Sestak et al., 1971).

LAI =

\section{Leaf area duration (LAD)}

Leaf area duration is the integral of leaf area index over a growth period (Watson, 1952). LAD for various growth periods was worked 
out as per the formula of Power et al., (1967) and expressed in days.

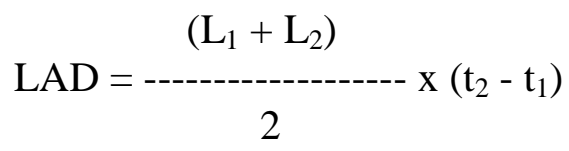

Where,

$\mathrm{L}_{1}=\mathrm{LAI}$ at time $\mathrm{t}_{1}, \mathrm{~L}_{2}=\mathrm{LAI}$ at time $\mathrm{t}_{2}, \mathrm{t}_{2}-\mathrm{t}_{1}=$ Time interval in days

The data recorded on various parameters were subjected to Fisher's method of analysis of variance and interpretation of the data as given by Gomez and Gomez (1984). The level of significance used in ' $\mathrm{F}$ ' and ' $\mathrm{t}$ ' test was $\mathrm{P}=$ 0.05. Critical difference (CD) values were calculated where the ' $F$ ' test was found significant. In case of non-significant effects, values of standard error of means alone were presented in Tables. The mean value of sub sub plot and interaction were separately subjected to Ducan's multiple range test (DMRT) using the corresponding error mean sum of squares and degrees of freedom values.

\section{Results and Discussion}

Total dry matter production of clusterbean, genotypes was found significant at all growth stages except at 30 DAS (Table: 1). Gaurishankar - 9 recorded significantly higher total dry matter production at $60(21.12 \mathrm{~g}$ plant $\left.^{-1}\right), 90$ DAS (23.99 $\left.\mathrm{g} \mathrm{plant}^{-1}\right)$ and at harvest $\left(26.18 \mathrm{~g} \mathrm{plant}^{-1}\right)$ when compared to the genotype $\mathrm{HG}-365$. At 60, 90 DAS and at harvest, at spacing of $45 \times 15 \mathrm{~cm}$ recorded significantly higher total dry matter production $\left(20.30,23.37\right.$ and $27.06 \mathrm{~g} \mathrm{plant}^{-1}$ respectively) when compared to at spacing of $30 \times 10 \mathrm{~cm}\left(17.10,19.97\right.$ and $23.18 \mathrm{~g} \mathrm{plant}^{-1}$ respectively). At 30 DAS, Bradyrhizobium + PSB + AM fungi recorded significantly higher total dry matter production $(3.47 \mathrm{~g}$ plant $^{-1}$ ) and was found on par with Bradyrhizobium and PSB alone (3.33 and $3.47 \mathrm{~g} \mathrm{plant}^{-1}$ respectively). At 60, 90 DAS and at harvest, Bradyrhizobium + PSB + AM fungi was recorded significantly higher total dry matter production $(21.30,24.19$ and 27.06 $\mathrm{g}$ plant $^{-1}$ respectively) when compared to other treatments. Differences in dry matter production were mainly because of various physiological factors specially photosynthetic capacity and reproductive phase. The photosynthetic activity of plants at various stages of crop growth can be assessed through leaf area, dry matter accumulation in leaves which in turn influence photosynthetic ability of plant and its performance finally on yield of any crop. The dry matter accumulated in leaves of Gaurishankar - 9 was 11.1, 20.7, 28.5 and 10 per cent higher at 30, 60, 90 DAS and at harvest respectively over HG - 365. Dry matter accumulation in leaves of clusterbean genotypes were found significant at all growth stages. The genotype, Gaurishankar - 9 recorded significantly higher dry matter accumulation in leaves at 30 (1.89g plant $\left.{ }^{-1}\right), 60$ (7.71 g plant $\left.^{-1}\right), 90$ DAS $\left(7.02 \mathrm{~g} \mathrm{plant}^{-1}\right)$ and at harvest $\left(1.05 \mathrm{~g} \mathrm{plant}^{-1}\right)$ compared to genotype $\mathrm{HG}-365$. Dry matter accumulation in leaves significantly varied due to spacing levels at 60 and 90 DAS. At 60 and 90 DAS, at spacing of $45 \times 15 \mathrm{~cm}$ recorded significantly higher dry matter accumulation in leaves (7.30 and $6.54 \mathrm{~g} \mathrm{plant}^{-}$ ${ }^{1}$ respectively) compared to the spacing of 30 $\times 10 \mathrm{~cm}$. Bio- inoculant treatments had significant influence on dry matter accumulation in leaves at 60 and 90 DAS. At 60 and 90 DAS, Bradyrhizobium + PSB + AM fungi was recorded significantly higher dry matter accumulation in leaves (8.07 and $7.25 \mathrm{~g}$ plant $^{-1}$ respectively) over other treatments. Dry matter accumulation in the stem of clusterbean genotypes were found significant at all growth stages except at 30 DAS. Gaurishankar - 9 recorded significantly higher dry matter accumulation in the stem at 
60 (7.23 g plant $\left.{ }^{-1}\right), 90$ DAS (7.86 g plant $\left.^{-1}\right)$ and at harvest $\left(9.11 \mathrm{~g} \mathrm{plant}^{-1}\right)$ compared to genotype HG - 365 (5.97, 6.68 and $7.72 \mathrm{~g}$ plant $^{-1}$ respectively). The spacing had significant influence on dry matter accumulation in stem at all growth stages except at 30 DAS. At 60 and 90 DAS and harvest, at spacing of $45 \times 15 \mathrm{~cm}$ was recorded significantly higher dry matter accumulation in stem $(7.23,7.94$ and $9.06 \mathrm{~g}$ plant $^{-1}$ respectively) compared to spacing of $30 \times 10 \mathrm{~cm}$. Bio- inoculant treatments had significant influence on dry matter accumulation in stem at 60,90 DAS and at harvest and had non-significant effect on dry matter accumulation in stem at 30 DAS. At 60 DAS, Bradyrhizobium + PSB + AM fungi recorded significantly higher dry matter accumulation in stem (7.30 $\left.\mathrm{g} \mathrm{plant}^{-1}\right)$ when compared to other treatments.

At 90 DAS and at harvest Bradyrhizobium + PSB + AM fungi was recorded significantly higher dry matter accumulation in stem (7.88 and $9.05 \mathrm{~g} \mathrm{plant}^{-1}$ respectively) and was found on par with Bradyrhizobium (7.26 and 8.42g plant $^{-1}$ respectively). Gaurishankar - 9 recorded significantly higher dry matter accumulation in reproductive parts at $60(6.17$ g plant $\left.{ }^{-1}\right), 90$ DAS (9.11 g plant ${ }^{-1}$ ) and at harvest (16.03 $\left.\mathrm{g} \mathrm{plant}^{-1}\right)$ compared to the genotype $\mathrm{HG}-365$. Dry matter accumulation in reproductive parts varied significantly due to influence of spacing treatments. At 60, 90 DAS and at harvest, spacing of $45 \times 15 \mathrm{~cm}$ recorded significantly higher dry matter accumulation in reproductive parts at $60(5.78$ g plant $\left.{ }^{-1}\right), 90$ DAS $\left(8.89 \mathrm{~g}_{\text {plant }}{ }^{-1}\right)$ and at harvest (15.56 $\left.\mathrm{g} \mathrm{plant}^{-1}\right)$ compared to spacing of $30 \times 10 \mathrm{~cm}$. Bio- inoculant treatments had significant effect on dry matter accumulation in reproductive parts at 60,90 DAS and at harvest. At 60 DAS, Bradyrhizobium + PSB + AM fungus was recorded significantly higher dry matter accumulation in reproductive parts $\left(5.93 \mathrm{~g} \mathrm{plant}^{-1}\right)$ over other treatments. At 90 DAS, Bradyrhizobium + PSB + AM fungus was recorded significantly higher dry matter accumulation in reproductive parts $\left(9.05 \mathrm{~g} \mathrm{plant}^{-1}\right)$ over other treatments and this was on par with Bradyrhizobium (8.42 $\left.\mathrm{g} \mathrm{plant}^{-1}\right)$. At harvest Bradyrhizobium + PSB + AM fungus was recorded significantly higher dry matter accumulation in reproductive parts $(16.97 \mathrm{~g}$ plant $^{-1}$ ) over other treatments. The higher dry matter accumulation might be due to higher leaf area, leaf area index and leaf area duration. Leaf area which is also an important part of the plant that determines the photosynthetic ability growth and dry matter production.

Leaf area of clusterbean varied significantly due to genotypes at all growth stages (Table: 3 ). The genotype, Gaurishankar - 9 recorded significantly higher leaf area at 30 (189.72 $\mathrm{cm}^{2}$ plant $\left.^{-1}\right), 60\left(670.38 \mathrm{~cm}^{2}\right.$ plant $\left.^{-1}\right), 90$ DAS $\left(448.19 \mathrm{~cm}^{2}\right.$ plant $\left.^{-1}\right)$ and at harvest $(99.76$ $\mathrm{cm}^{2}$ plant ${ }^{-1}$ ) compared to genotype HG - 365 $\left(158.38,517.49,312.85\right.$ and $81.19 \mathrm{~cm}^{2}$ plant $^{-1}$ respectively). The spacing of $45 \times 15 \mathrm{~cm}$ recorded significantly higher leaf area at 30 $\left(184.61 \mathrm{~cm}^{2}\right.$ plant $\left.^{-1}\right), 60\left(642.82 \mathrm{~cm}^{2}\right.$ plant $\left.^{-1}\right)$, $90\left(412.65 \mathrm{~cm}^{2}\right.$ plant $\left.^{-1}\right)$ and at harvest $\left(96.11 \mathrm{~cm}^{2}\right.$ plant $\left.^{-1}\right)$ when compared to at spacing of $30 \times 10 \mathrm{~cm}(163.49,545.05$, 348.38 and $84.85 \mathrm{~cm}^{2}$ plant $^{-1}$ respectively).

Bio-inoculant treatments had significant effect on leaf area at all growth stages except at 30 DAS. At 60, 90 DAS and at harvest Bradyrhizobium + PSB + AM fungi recorded significantly higher leaf area (704.28, 439.26 and $103.88 \mathrm{~cm}^{2}$ plant ${ }^{-1}$ respectively) compared to other treatments. Between the genotypes of clusterbean, Gaurishankar - 9 recorded significantly higher leaf area index at 30 (0.44), 60 (1.55), 90 DAS (1.03) and at harvest (0.24) when compared to the genotype HG - $365 \quad(0.37, \quad 1.21,0.69$ and 0.19 respectively). 
Table.1 Total dry matter accumulation per plant and dry matter accumulation in reproductive of clusterbean at different growth stages as influenced by genotypes, plant density and bio-

inoculants

\begin{tabular}{|l|l|l|l|l|l|l|l|}
\hline Treatments & $\begin{array}{l}\text { Total dry } \\
\text { matter } \\
\text { production } \\
\text { at 30 DAS }\end{array}$ & $\begin{array}{l}\text { Total dry } \\
\text { matter } \\
\text { production } \\
\text { at 60 DAS }\end{array}$ & $\begin{array}{l}\text { Total dry } \\
\text { matter } \\
\text { production } \\
\text { at 90 DAS }\end{array}$ & $\begin{array}{l}\text { Total dry } \\
\text { matter } \\
\text { production at } \\
\text { harvest }\end{array}$ & $\begin{array}{l}\text { Dry matter } \\
\text { accumulatio } \\
\text { n in } \\
\text { reproductive } \\
\text { parts } \\
\text { (60DAS) }\end{array}$ & $\begin{array}{l}\text { Dry matter } \\
\text { accumulation } \\
\text { in } \\
\text { reproductive } \\
\text { parts } \\
\text { (90DAS) }\end{array}$ & $\begin{array}{l}\text { Dry matter } \\
\text { accumulation } \\
\text { in } \\
\text { reproductive } \\
\text { parts } \\
\text { harvest }\end{array}$ \\
at \\
\hline Genotype
\end{tabular}

Table. 2 Dry matter accumulation in leaves and stem of clusterbean at different growth stages as influenced by genotypes, plant density and bio-inoculants

\begin{tabular}{|c|c|c|c|c|c|c|c|c|}
\hline Treatments & $\begin{array}{l}\text { Dry matter } \\
\text { accumulati } \\
\text { on in } \\
\text { leaves } \\
\text { (30DAS) }\end{array}$ & $\begin{array}{c}\text { Dry matter } \\
\text { accumulati } \\
\text { on in } \\
\text { leaves } \\
\text { (60DAS) }\end{array}$ & $\begin{array}{c}\text { Dry matter } \\
\text { accumulati } \\
\text { on in } \\
\text { leaves } \\
\text { (90DAS) }\end{array}$ & $\begin{array}{c}\text { Dry } \\
\text { matter } \\
\text { accumulat } \\
\text { ion in } \\
\text { leaves } \\
\text { (Harvest) }\end{array}$ & $\begin{array}{c}\text { Dry } \\
\text { matter } \\
\text { accumulat } \\
\text { ion in } \\
\text { stem } \\
\text { (30DAS) }\end{array}$ & $\begin{array}{c}\text { Dry } \\
\text { matter } \\
\text { accumulat } \\
\text { ion in } \\
\text { stem } \\
\text { (60DAS) }\end{array}$ & $\begin{array}{l}\text { Dry matter } \\
\text { accumulati } \\
\text { on in stem } \\
\text { (90DAS) }\end{array}$ & $\begin{array}{c}\text { Dry matter } \\
\text { accumulati } \\
\text { on in stem } \\
\text { at harvest }\end{array}$ \\
\hline \multicolumn{9}{|l|}{ Genotype } \\
\hline HG-365 & $1.69 \mathrm{~b}$ & $6.14 \mathrm{~b}$ & $5.03 \mathrm{~b}$ & $0.95 b$ & $1.28 \mathrm{a}$ & $5.97 \mathrm{~b}$ & $6.68 \mathrm{~b}$ & $7.72 b$ \\
\hline Gaurishankar-9 & $1.89 \mathrm{a}$ & $7.71 \mathrm{a}$ & $7.02 \mathrm{a}$ & $1.05 \mathrm{a}$ & $1.67 \mathrm{a}$ & $7.23 a$ & $7.86 a$ & $9.11 \mathrm{a}$ \\
\hline S. Em \pm & 0.03 & 0.20 & 0.14 & 0.01 & 0.13 & 0.11 & 0.01 & 0.07 \\
\hline \multicolumn{9}{|l|}{ Spacing } \\
\hline $30 \times 10 \mathrm{~cm}$ & $1.74 \mathrm{a}$ & $6.56 \mathrm{~b}$ & $5.51 \mathrm{~b}$ & $0.97 \mathrm{a}$ & $1.45 \mathrm{a}$ & $5.98 \mathrm{~b}$ & $6.60 \mathrm{~b}$ & $7.77 \mathrm{~b}$ \\
\hline $45 \times 15 \mathrm{~cm}$ & $1.84 \mathrm{a}$ & $7.30 \mathrm{a}$ & $6.54 \mathrm{a}$ & $1.03 \mathrm{a}$ & $1.50 \mathrm{a}$ & $7.23 a$ & $7.94 \mathrm{a}$ & $9.06 \mathrm{a}$ \\
\hline S. Em \pm & 0.06 & 0.13 & 0.16 & 0.02 & 0.05 & 0.31 & 0.28 & 0.29 \\
\hline \multicolumn{9}{|l|}{ Bio inoculants } \\
\hline Bradyrhizobium & $1.87 \mathrm{a}$ & $7.21 \mathrm{~b}$ & $6.19 \mathrm{~b}$ & $0.99 \mathrm{a}$ & $1.47 \mathrm{a}$ & $6.59 \mathrm{~b}$ & $7.26 \mathrm{~b}$ & $8.42 \mathrm{ab}$ \\
\hline PSB & $1.75 \mathrm{ab}$ & $6.37 \mathrm{c}$ & $5.55 \mathrm{c}$ & $1.04 \mathrm{a}$ & $1.44 \mathrm{a}$ & $6.29 \mathrm{~b}$ & $6.96 \mathrm{~b}$ & $8.13 b$ \\
\hline AM fungi & $1.61 \mathrm{ab}$ & $6.07 \mathrm{c}$ & $5.10 \mathrm{c}$ & $0.93 \mathrm{a}$ & $1.46 \mathrm{a}$ & $6.23 \mathrm{~b}$ & $6.98 \mathrm{~b}$ & $8.06 \mathrm{~b}$ \\
\hline $\begin{array}{l}\text { Bradyrhizobium + } \\
\text { PSB + AM fungi }\end{array}$ & $1.93 \mathrm{a}$ & $8.07 \mathrm{a}$ & $7.25 \mathrm{a}$ & $1.04 \mathrm{a}$ & $1.54 \mathrm{a}$ & $7.30 \mathrm{a}$ & $7.88 \mathrm{a}$ & $9.05 \mathrm{a}$ \\
\hline S. Em \pm & 0.07 & 0.21 & 0.21 & 1.03 & 0.06 & 0.18 & 0.24 & 0.25 \\
\hline
\end{tabular}


Table.3 Leaf area, leaf area index and leaf area duration of clusterbean at different growth stages as influenced by genotypes, plant density and bio-inoculants

\begin{tabular}{|c|c|c|c|c|c|c|c|c|c|c|c|}
\hline Treatments & $\begin{array}{l}\text { Leaf area } \\
\text { (30DAS) }\end{array}$ & $\begin{array}{l}\text { Leaf area } \\
\text { (60DAS) }\end{array}$ & $\begin{array}{l}\text { Leaf area } \\
\text { (90DAS) }\end{array}$ & $\begin{array}{l}\text { Leaf area } \\
\text { (Harvest) }\end{array}$ & $\begin{array}{l}\text { Leaf area } \\
\text { index } \\
(30 D A S)\end{array}$ & $\begin{array}{l}\text { Leaf area } \\
\text { index } \\
(60 D A S)\end{array}$ & $\begin{array}{c}\text { Leaf area } \\
\text { index } \\
\text { (90DAS) }\end{array}$ & $\begin{array}{c}\text { Leaf area } \\
\text { index } \\
\text { (Harvest) }\end{array}$ & $\begin{array}{c}\text { Leaf area } \\
\text { duration } \\
(30- \\
\text { 60DAS }) \\
\end{array}$ & $\begin{array}{c}\text { Leaf area } \\
\text { duration } \\
(60- \\
\text { 90DAS }) \\
\end{array}$ & $\begin{array}{c}\text { Leaf area } \\
\text { duration } \\
\text { (90 DAS - } \\
\text { Harvest) }\end{array}$ \\
\hline \multicolumn{12}{|l|}{ Genotype } \\
\hline HG-365 & $158.38 \mathrm{~b}$ & $517.49 b$ & $312.85 b$ & $81.19 \mathrm{~b}$ & $0.37 \mathrm{~b}$ & $1.21 \mathrm{~b}$ & $0.69 \mathrm{~b}$ & $0.19 b$ & $23.81 \mathrm{~b}$ & $28.57 b$ & $13.22 b$ \\
\hline Gaurishankar-9 & $189.72 \mathrm{a}$ & $670.38 \mathrm{a}$ & $448.19 a$ & $99.76 \mathrm{a}$ & $0.44 \mathrm{a}$ & $1.55 \mathrm{a}$ & $1.03 \mathrm{a}$ & $0.24 \mathrm{a}$ & $29.88 \mathrm{a}$ & $38.79 \mathrm{a}$ & $19.08 \mathrm{a}$ \\
\hline S. Em \pm & 5.01 & 17.09 & 8.45 & 1.62 & 0.01 & 0.05 & 0.02 & 0.004 & 0.73 & 1.61 & 0.32 \\
\hline \multicolumn{12}{|l|}{ Spacing } \\
\hline $30 \times 10 \mathrm{~cm}$ & $163.49 \mathrm{~b}$ & $545.05 b$ & $348.38 \mathrm{~b}$ & $84.85 \mathrm{~b}$ & $0.54 \mathrm{a}$ & $1.81 \mathrm{a}$ & $1.12 \mathrm{a}$ & $0.28 \mathrm{a}$ & $35.30 \mathrm{a}$ & $43.89 \mathrm{a}$ & $21.01 \mathrm{a}$ \\
\hline $45 \times 15 \mathrm{~cm}$ & $184.61 \mathrm{a}$ & $642.82 \mathrm{a}$ & $412.65 \mathrm{a}$ & $96.11 \mathrm{a}$ & $0.27 \mathrm{~b}$ & $0.95 \mathrm{~b}$ & $0.61 \mathrm{~b}$ & $0.14 \mathrm{~b}$ & $18.39 \mathrm{~b}$ & $23.48 \mathrm{~b}$ & $11.28 \mathrm{~b}$ \\
\hline S. Em \pm & 1.83 & 16.27 & 8.71 & 1.18 & 0.01 & 0.05 & 0.04 & 0.01 & 0.66 & 1.03 & 0.82 \\
\hline \multicolumn{12}{|l|}{ Bio inoculants } \\
\hline Bradyrhizobium & $175.85 \mathrm{a}$ & $620.94 b$ & $393.02 b$ & $91.48 \mathrm{~b}$ & $0.41 \mathrm{a}$ & $1.47 \mathrm{~b}$ & $0.93 a$ & $0.22 \mathrm{ab}$ & $28.27 \mathrm{~b}$ & $36.15 \mathrm{a}$ & $17.17 \mathrm{a}$ \\
\hline PSB & $169.58 \mathrm{a}$ & $526.54 \mathrm{c}$ & $348.20 \mathrm{c}$ & $84.99 b c$ & $0.40 \mathrm{a}$ & $1.21 \mathrm{c}$ & $0.76 b$ & $0.20 \mathrm{bc}$ & $24.20 \mathrm{c}$ & $29.62 b$ & $14.41 \mathrm{~b}$ \\
\hline AM fungi & $170.10 \mathrm{a}$ & $523.99 \mathrm{c}$ & $341.60 \mathrm{c}$ & $81.56 \mathrm{c}$ & $0.40 \mathrm{a}$ & $1.22 \mathrm{c}$ & $0.75 b$ & $0.19 \mathrm{c}$ & $24.31 \mathrm{c}$ & $29.51 \mathrm{~b}$ & $14.05 \mathrm{~b}$ \\
\hline $\begin{array}{l}\text { Bradyrhizobium }+ \\
\text { PSB + AM fungi }\end{array}$ & $180.67 \mathrm{a}$ & $704.28 \mathrm{a}$ & $439.26 \mathrm{a}$ & $103.88 \mathrm{a}$ & $0.42 \mathrm{a}$ & $1.62 \mathrm{a}$ & $1.01 \mathrm{a}$ & $0.24 \mathrm{a}$ & $30.60 \mathrm{a}$ & $39.45 \mathrm{a}$ & $18.95 \mathrm{a}$ \\
\hline S. Em \pm & 4.90 & 17.79 & 13.19 & 2.51 & 0.01 & 0.04 & 0.05 & 0.01 & 0.74 & 1.46 & 0.81 \\
\hline
\end{tabular}

The spacing of $30 \times 10 \mathrm{~cm}$ realized significantly higher leaf area index at 30 (0.54), 60(1.81), 90 DAS (1.12) and at harvest (0.28) when compared to the spacing of $45 \times 15 \mathrm{~cm}(0.27$, $0.95,0.61$ and 0.14 respectively). Bio- inoculant treatments had significant effect on leaf area index at all growth stages except at 30 DAS. At 60 DAS and at harvest, Bradyrhizobium + PSB + AM fungi recorded significantly higher leaf area (1.62 and 0.24 respectively) when compared to other treatments. At 90 DAS, Bradyrhizobium + PSB + AM fungi recorded significantly higher leaf area (1.01) and was found on par with Bradyrhizobium alone (0.93) over other treatments. The LAI is often used as vital indicator of plant growth for evaluating assimilation, transpiration rates and is a major factor for determining the solar radiation interception, photosynthesis and therefore yield of clusterbean. Gaurishankar - 9 recorded higher LAI at all growth stages compared to HG - 365 . Similarly, Gaurishankar - 9 realised higher LAD (29.8 days) during 30 - 60 DAS than HG 365 (23.8 days). Total number of nodules and nodule dry weight recorded higher in case of genotype Gaurishankar - 9 (Guriqbal et al., 2012 in urdbean). These indicated that genotype Gaurishankar - 9 produced higher dry matter as compared to HG - 365 (Table 22 and Fig. 6).
Similar results of higher dry matter production were also reported by Ashwathanarayana (2014) in clusterbean and Madhu (2013) in mungbean.

Between the genotypes of clusterbean, Gaurishankar - 9 recorded significantly higher leaf area duration 30- 60 DAS (29.88 days), 6090 DAS (38.79 days) and 90 - harvest (19.08 days) when compared to the genotype $\mathrm{HG}-$ 365. The spacing of $30 \times 10 \mathrm{~cm}$ recorded significantly higher leaf area duration at 30-60 DAS 35.30 days), 60- 90 DAS (43.89 days) and 90 - harvest (21.01 days) compared to a spacing of $45 \times 15 \mathrm{~cm}$. Bio- inoculant treatments had significant effect on leaf area duration at 30- 60 DAS, 60- 90 DAS and 90 - harvest. At 30- 60, Bradyrhizobium + PSB + AM fungi recorded significantly higher leaf area duration (30.60 days). At 60- 90 DAS Bradyrhizobium + PSB + AM fungi recorded significantly higher leaf area duration (39.45 days) and this was found on par with Bradyrhizobium alone (36.15 days). At 90 DAS - harvest, same trend was followed as that of $60-90$ DAS.

Gaurishankar - 9 recorded higher total dry matter production per plant, dry matter accumulation in different plant parts like leaves, stem and reproductive parts, leaf area and leaf 
area duration at different growth stages. The spacing level of $45 \times 15 \mathrm{~cm}$ higher total dry matter production per plant, dry matter accumulation in different plant parts like leaves, stem and reproductive parts, leaf area and leaf area duration at different growth stages compared to spacing level of $30 \times 10 \mathrm{~cm}$. Application Bradyrizhobium + PSB + AM fungi recorded higher total dry matter production per plant, dry matter accumulation in different plant parts like leaves, stem and reproductive parts, leaf area and leaf area duration at different growth stages of crop compared to the other treatments.

\section{References}

Anonymous, 2013, Ministry of Agriculture, Govt. of India.secy-agri@ nic.in

Ashwathanarayana, S. T., 2014, Intercropping of pigeonpea [Cajanus cajan (L.) Millsp.] with gum guar genotypes [Cyamopsis tetragonoloba (L.) Taub.] in different row proportions. M. Sc. (Agri.) Thesis, Univ. Agril. Sci., Raichur, India.

Choudhary, R. S., Yadav, R. S. and Amin, A., 2014, Productivity and economics of clusterbean (Cyamopsis tetragonoloba) as influenced by phosphorus fertilization and biofertilizers in western Rajasthan. Ann. Agric. Res. New Series, 35(1): 62-64.

Gomez, K. A. and Gomez, A. A., 1984, Statistical Procedure for Agricultural Research. John Wiley and Sons, New Delhi, p. 680.

Guriqbal, S., Hari, R. K. K., Sekhon, H. S., Gill and Veena, K., 2012, Effect of time of planting on nodulation growth and seed yield of kharif urdbean genotypes. $J$. Food Leg., 25 (2):125.127.

Khandelwal, R., Choudhary, S. K., Khangarot, S. S., Jat, M. K. and Singh, P., 2012, Effect of inorganic and bio-fertilizers on productivity and nutrients uptake in cowpea [Vigna unguiculata (L.) Walp]. Legume Res., 35 (3): 235 -238.

Madhu, G., 2013, Response of mungbean (Vigna radiata L. Wilczek) genotypes to dates of sowing and foliar nutition in kharif season. M. Sc. (Agri.) Thesis, Univ. Agril. Sci., Dharwad, India.

Power, J. E., Wills, W. O., Granes, D. L. and Reichman, G. A., 1967, Effect of soil temperature, phosphorus and plant age on growth analysis of barley. J. Agron., 59: 231-234.

Rathore, V. S., Singh, J. P., Soni, M. L., Yadava, N. D. and Beniwal, R. K., 2007, Productivity, quality and resource utilization by clusterbean (Cyamopsis tetragonoloba) as influenced by nutrient management. Indian J. Agron., 52 (3): 243-246.

Sestak, Z., Castsky, J. and Jarvis, P. G., 1971, Plant photosynthetic production. Mannual of methods (Ed.). W. JUNK, N. V., publication. The Hughus, 343-381.

Vivekanandan, A. S., Gunasena, H. P. M. and Sivananyagam, T., 1972, Statistical evaluation of accuracy of three techniques used in estimation of leaf area of crop plants. Indian J. Agric. Sci., 42: 847-860.

Watson, D. J., 1952. The physiological basis of variation in yield. Advances in Agron, 4: 101-145.

\section{How to cite this article:}

Shilpa V. Chogatapur and Chandranath, H.T. 2017. Dry matter Production and Partitioning of Cluster Bean (Cyamopsis tetragonaloba (L). taub.) Genotypes (Gum) as Influenced by Plant Density and Bio-Inoculants. Int.J.Curr.Microbiol.App.Sci. 6(12): 1797-1803. doi: https://doi.org/10.20546/ijcmas.2017.612.203 